\title{
Traffic Network and Optimization a Future Subscriber's Mobile Telecom Operator in Train
}

\section{Allami J*, Ez-Zahraouy $\mathrm{H}$ and Benyoussef $\mathrm{A}$}

Laboratory of Magnetism and High Energy Physics (LMPHE), Faculty of Sciences, Rabat, Morocco

\begin{abstract}
In this work, we studied the behavior and mobility of telecom subscribers into a train for now and predict a future telecom movement subscriber and have a perfect resources data signal of an operator mobile telecom, we used a deterministic and a probabilistic method. the train in our model example travels through a cellular network and passed into four zones $(Z 1, Z 2, Z 3$ and Z4), each one is characterized by: topography, numbers of the cellular network, types of network (GSM, GPRS, UMTS ...), numbers of subscribers, types of subscribers(staffs, students, workers and others), numbers of operators (Operator 1, Operator 2 and Operator 3). We have studied statistics in deterministic and probabilistic vision of traffic telecom; the model used is approach to vehicular traffic model.
\end{abstract}

Keywords: Network planning; Cellular network traffic; Mobility; Cellular automata; Deterministic model; Probabilistic model; Telecom mobile; Vehicular technology

\section{Introduction}

We noticed that in train the telephone company cannot meet the needs of telecom subscribers (GSM, UMTS, GPRS ..) which carry a train in the path movement Kenitra-Sale-Rabat-Casa, for this we have proposed a model statistical study of the real traces of subscribers of different telecom operator (Operator1, Operator2 and Operator3) for data needed on subscribers train in this route cities: Kenitra ----> Sale ----> Rabat-- -> Casa. We study a real telecom subscribers traces and data of telecom operators in Morocco, and make a simulation by Matlab software by introducing the density necessary scientific and technical vision in the mobile telecom traffic.

The traffic and mobility model [1-6], is used in several operations of the cellular network, we have studied statistics for the maximum information from a subscriber to properly estimate and control the flow of a telecom operator in a given area, the study contains (region, topography, operator, types of network (GSM, GPRS, UMTS), speed, number of subscribers). for example : a subscriber telecom moves from Kenitra(Z1) $---\rightarrow$ Sale(Z2) $--\rightarrow$ Rabat(Z3) $--\rightarrow$ Casa(Z4) (areas or cities train ride) with different characteristics of each area. This model is based on the concept of cellular automata [7-9] which uses the density of subscribers based on hours $\rho(t)$, this study does help operators to adjust the zones (visited, size, type, frequency of travel, fields, speed), allowing the network to control, plan and optimize a future movement of a subscriber telecom operator. By introducing the parameters required by inspiring model cellular automata (CA) $[7,8,10]$ and traffic modeling $[11,12]$, traffic accident $[13,14]$, high way junctions on the neck [15], vehicle acceleration [16]. This study uses realistic conditions of the telecommunication network types with GSM, GPRS, UMTS and WIMAX (for different areas). We have studied the case of a mobile field studies with statistics (size, topography, number of operators, numbers of people, behavior, operator telecom subscribers).

We study a mathematical technique [3], simulation [4] and operating traces [17-19] which are based on the statement of assumptions and approximations use planning, mobility, network traffic modeling mobile (GSM, GPRS, UMTS ...) and also on the simulation requires very long computation time.

In our method all traces of actual (realistic model) and also based on the exploitation of traces [20] which allows to use the data to easily extract and exploit networks of trace data using a sample population of subscribers model $[21,22]$ which gives a user profile with its movement in a deterministic case [23-27], to generalize if one follows a probability that can predict future movements of subscribers (all parameters of network traces), network planning, study area, extract (raw file) traces GSM/GPRS with the mobility of a subscriber (cell area Z1, Z2, Z3 and Z4) to properly optimize and estimate a future mobility subscribers of an operators mobile telecom (Figure 1).

\section{Major Transitions}

It carries the traces collected by a technique based on the exploitation of the data measured using the methods [28-34]. We use the modeling levels of cells to calculate the transition of moving from a current state to future state $k(x, y)$ of a BTS: $x_{k+1}=\theta_{k} x_{k}+w_{k}$ with $w_{k}$ : correction parameter subscribers - BTS $\theta_{k}$ : parameter relating $x_{k}$ to future $x_{k+1}$

$$
\left[\begin{array}{l}
x \\
v_{x} \\
y \\
v_{y}
\end{array}\right]_{k+1}=\left[\begin{array}{llll}
1 & t & 0 & 0 \\
0 & 1 & 0 & 0 \\
0 & 0 & 1 & t \\
0 & 0 & 0 & 1
\end{array}\right]_{t}\left[\begin{array}{l}
x \\
v_{x} \\
y \\
v_{y}
\end{array}\right]_{k}+\left[\begin{array}{l}
w_{1} \\
w_{2} \\
w_{3} \\
w_{4}
\end{array}\right]_{k}
$$

$(x, y)$ : coordinates of a cell BTS

$\left(v_{x}, v_{y}\right)$ : speed between 2 cells.

It incorporates the concept area by modeling in the area.

\section{Deterministic Case}

We choose a transition parameter, chatting in the day time interval:

*Corresponding author: Allami J, Laboratory of Magnetism and High Energy Physics (LMPHE), Faculty of Sciences, Rabat, Morocco, Tel: + 212 (0) 5377718 34/35/38; E-mail: jaouad.allami@gmail.com

Received October 28, 2014; Accepted May 30, 2015; Published June 06, 2015

Citation: Allami J, Ez-Zahraouy H, Benyoussef A (2015) Traffic Network and Optimization a Future Subscriber's Mobile Telecom Operator in Train. J Generalized Lie Theory Appl 9: 219. doi: 10.4172/1736-4337.1000219

Copyright: ( $) 2015$ Allami J, et al. This is an open-access article distributed under the terms of the Creative Commons Attribution License, which permits unrestricted use, distribution, and reproduction in any medium, provided the original author and source are credited. 
Citation: Allami J, Ez-Zahraouy H, Benyoussef A (2015) Traffic Network and Optimization a Future Subscriber's Mobile Telecom Operator in Train. J Generalized Lie Theory Appl 9: 219. doi: 10.4172/1736-4337.1000219

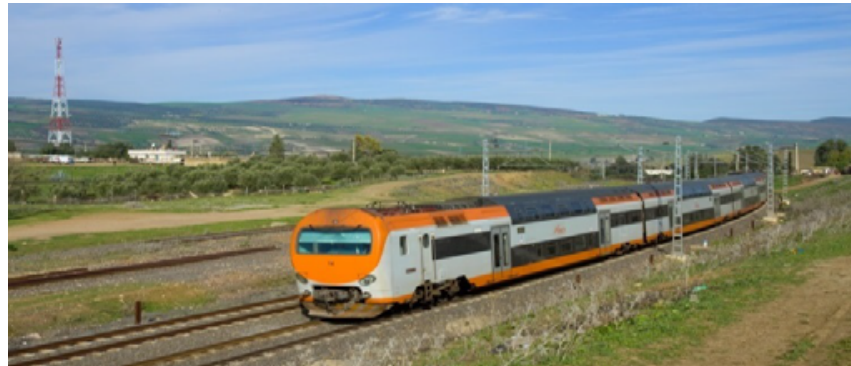

Figure 1: Mobile Network Train Kenitra-Sale-Rabat-Casa in Morocco.

$\left(x_{k+1}\right)_{z}=T\left(\theta_{k} x_{k}+w_{k}\right)_{z}$ whichhas a matrix of time:

$$
\left[\begin{array}{l}
\left(x_{k+1}\right)_{z_{1}} \\
\left(x_{k+1}\right)_{z_{2}} \\
\left(x_{k+1}\right)_{z_{3}} \\
\left(x_{k+1}\right)_{z_{4}}
\end{array}\right]=\left[\begin{array}{cccc}
T_{1} & T_{21} & T_{31} & T_{41} \\
T_{12} & T_{2} & T_{32} & T_{42} \\
T_{13} & T_{23} & T_{3} & T_{43} \\
T_{14} & T_{24} & T_{34} & T_{4}
\end{array}\right]\left[\begin{array}{l}
\left(\theta_{k} x_{k}+w_{k}\right)_{z_{1}} \\
\left(\theta_{k} x_{k}+w_{k}\right)_{z_{2}} \\
\left(\theta_{k} x_{k}+w_{k}\right)_{z_{3}} \\
\left(\theta_{k} x_{k}+w_{k}\right)_{z_{4}}
\end{array}\right]
$$

With: $T_{1}, T_{2}, T_{3}, T_{4}$ : time slots of stationary in $Z_{1}, Z_{2}, Z_{3}, Z_{4}$.

$T_{12}, T_{13}, T_{14}, T_{21}, T_{23}, T_{24}, T_{31}, T_{32}, T_{34}, T_{41}, T_{42}, T_{43}$ : time of transition from one area to another in the Train.

$$
T_{14}=T_{12}+T_{2}+T_{23}+T_{3}+T_{34} ; T_{41}=T_{43}+T_{3}+T_{32}+T_{2}+T_{21} .
$$

Case $z_{1}:\left(x_{k+1}\right)_{z_{1}}=T_{1}\left[\left(\theta_{k} x_{k}+w_{k}\right)_{z_{1}}\right]+T_{21}\left[\left(\theta_{k} x_{k}+w_{k}\right)_{z_{2}}\right]+T_{3 !}\left[\left(\theta_{k} x_{k}+w_{k}\right)_{z_{3}}\right]+T_{41}\left[\left(\theta_{k} x_{k}+w_{k}\right)_{z_{4}}\right]$

that describes the future state of a subscriber who comes from the same area or different area. : subscriber stationary in $z_{1}$

$T_{21}$ : subscriber moves from $z_{2}$ to $z_{1}$ in the Train.

This method is deterministic [22] means the subscriber $T_{1}, T_{21}, T_{31}, T_{41}$ must necessarily going to $z_{1}$, which does not produce the actual movement of a subscriber.

To make the model realistic, we introduce probability parameters.

\section{Probabilistic Method}

$$
\left[\begin{array}{l}
\left(x_{k+1}\right)_{z_{1}} \\
\left(x_{k+1}\right)_{z_{2}} \\
\left(x_{k+1}\right)_{z_{3}} \\
\left(x_{k+1}\right)_{z_{4}}
\end{array}\right]=\left[\begin{array}{cccc}
T_{1} & T_{21} & T_{31} & T_{41} \\
T_{12} & T_{2} & T_{32} & T_{42} \\
T_{13} & T_{23} & T_{3} & T_{43} \\
T_{14} & T_{24} & T_{34} & T_{4}
\end{array}\right]\left[\begin{array}{cccc}
p_{1} & p_{2} & p_{3} & p_{4} \\
p_{12} & p_{21} & p_{31} & p_{41} \\
p_{13} & p_{23} & p_{32} & p_{42} \\
p_{14} & p_{24} & p_{34} & p_{43}
\end{array}\right]\left[\begin{array}{l}
\left(\theta_{k} x_{k}+w_{k}\right)_{z_{1}} \\
\left(\theta_{k} x_{k}+w_{k}\right)_{z_{2}} \\
\left(\theta_{k} x_{k}+w_{k}\right)_{z_{3}} \\
\left(\theta_{k} x_{k}+w_{k}\right)_{z_{4}}
\end{array}\right]
$$

$p_{1,} p_{2,} p_{3,} p_{4}:$ probability of stationarity in $\mathrm{Z} 1, \mathrm{Z} 2, \mathrm{Z} 3, \mathrm{Z} 4$.

$p_{12,} p_{13,}, p_{14,} p_{21,} p_{23,} p_{24,}, p_{31,}, p_{32,} p_{34,} p_{41,} p_{42,} p_{43}:$ probability of transition from one area to another in the Train.

For $z_{1}$ :

$$
\begin{aligned}
&\left(x_{k+1}\right)_{21}=\left(T_{1} p_{1}+T_{21} p_{12}+T_{31} p_{13}+T_{41} p_{14}\right)\left(\theta_{k} x_{k}+w_{k}\right)_{21}+\left(T_{1} p_{2}+T_{21} p_{21}+T_{31} p_{23}+T_{41} p_{24}\right)\left(\theta_{k} x_{k}+w_{k}\right)_{21} \\
&\left(T_{1} p_{3}+T_{21} p_{31}+T_{31} p_{32}+T_{41} p_{34}\right)\left(\theta_{k} x_{k}+w_{k}\right)_{z_{3}}+\left(T_{1} p_{4}+T_{21} p_{41}+T_{31} p_{42}+T_{41} p_{43}\right)\left(\theta_{k} x_{k}+w_{k}\right)_{2_{4}} \\
&\left(x_{k+1}\right)_{z_{1}}=T_{1} p_{1}\left(\theta_{k} x_{k}+w_{k}\right)_{21}+T_{21} p_{21}\left(\theta_{k} x_{k}+w_{k}\right)_{z_{2}}+T_{31} p_{32}\left(\theta_{k} x_{k}+w_{k}\right)_{z_{3}}+T_{41} p_{43}\left(\theta_{k} x_{k}+w_{k}\right)_{z_{4}}
\end{aligned}
$$

Case: subscriber at the train is not mobile (zone 1) means $\left(x_{k+1}\right)_{z_{1}}=0$

Moving in the same region $\left(p_{1}>>p_{12}, p_{13}, p_{14}\right)$

means: $\left(x_{k+1}\right)_{z_{1}}=T_{1} p_{1}\left(\theta_{k} x_{k}+w_{k}\right)_{z_{1}}$
Subscriber is arrested in $z_{2}$ (train) $\left(p_{2} \gg p_{12}, p_{13}, p_{14}\right)$

Final equation: $\left(x_{k+1}\right)_{z}=S_{z}\left(\theta_{k} x_{k}+w_{k}\right)_{z}$

$S_{z}$ : matrix combination of two matrices $T_{z}$ and $p_{z}$.

By exploiting the raw materials measured in GSM/GPRS BTS which can serve several cells at the same time, so one or more cells may have the same coordinates of BTS.

The difficulty of attributing a subscriber to a cell is to determine its actual location; we choose a solution that sets the position according to its profile area.

\section{Zone Profile Creation}

An area of a network can be represented as a set of cells of different coordinated to define a profile of the region we define the profile of the cell $(x, y)$ using the following steps:

1) Data extraction of cells from each zone $c_{1}\left(x_{1}, y_{1}\right), c_{2}\left(x_{2}, y_{2}\right), c_{3}\left(x_{3}, y_{3}\right), c_{4}\left(x_{4}, y_{4}\right)$ (Figures 2 and 3 ).

Assign weight for each cell we add $p(w)$ that although the visible cell with the occupancy rate $c_{1}\left(x_{1}, y_{1}, p_{1}\right), c_{2}\left(x_{2}, y_{2}, p_{2}\right), c_{3}\left(x_{3}, y_{3}, p_{3}\right), c_{4}\left(x_{4}, y_{4}, p_{4}\right)$

Calculation of weighted coordinates

$c_{1}\left(x_{1} p_{1}, y_{1} p_{1}\right), c_{2}\left(x_{2} p_{2}, y_{2} p_{2}\right), c_{3}\left(x_{3} p_{3}, y_{3} p_{3}\right), c_{4}\left(x_{4} p_{4}, y_{4} p_{4}\right)$

The sum of the coordinates and weights

$\left(x_{1} p+x_{2} p+x_{3} p+x_{4} p ; y_{1} p+y_{2} p+y_{3} p+y_{4} p ; p\right)$

with $p$ : sum of all weights

In general for n cells: $x_{s}=\sum_{i=1}^{n} x_{n} p ; y_{s}=\sum_{i=1}^{n} y_{n} p ; p=\sum_{i=1}^{n} p_{n}$

Calculate the position of the zone $x_{z}=\frac{x_{s}}{;} y_{z}=\underline{y_{s}}$ $\left(x_{z}, y_{z}\right)$ gives the position of an area formed by a set of cells $p_{\text {of }}$ the sarne profile or a profile which gives the different rates of communication.

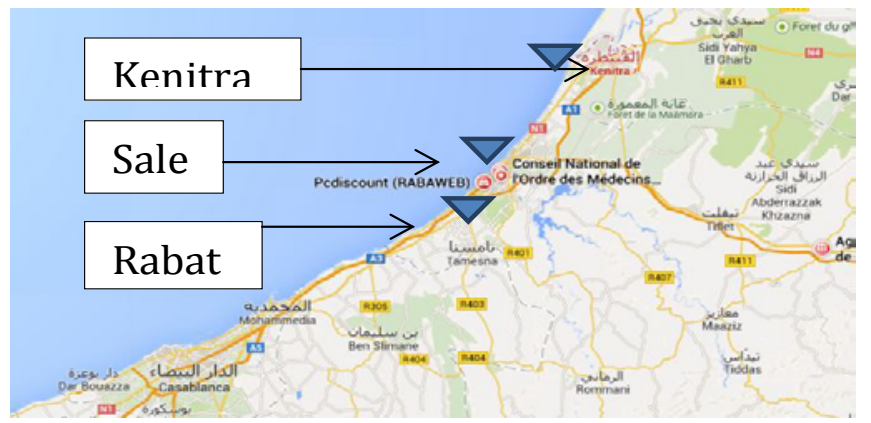

Figure 2: Data extraction of cells from each zone.

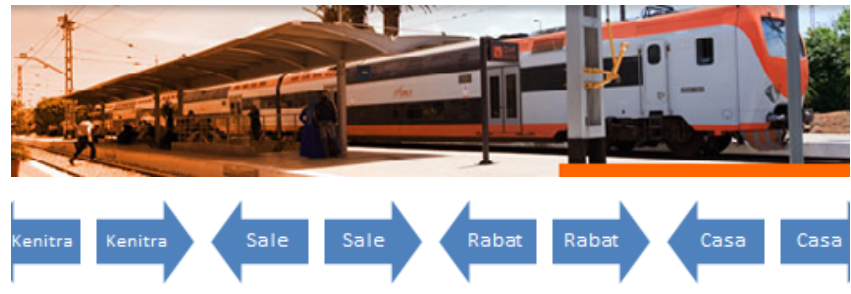

Figure 3: Train Ride Red Kenitra-Rabat-Sale-Casa in Morocco. 
$p:$ parameter which gives the speed of communication and the occupancy rate

\section{Integration a Density Parameter}

It incorporates density as a parameter to track the movement of a subscriber or a group of subscribers and at the same time whether that subscriber moves into train in an area with sufficient resources or not.

$\rho_{\neq 1}(t)=\frac{N_{a b(\neq 1)}(t)}{N_{r e s s}(\neq 1)}$

$N_{a b}$ : Number of subscribers per cell.

$N_{\text {ress }}$ : Number of resources provides a cell by the operator.

$\rho$ : Density mathematics in a cell $(\neq 1)$ in the train at a given time.

$t$ : Time slots

to generalize:

$\rho_{\neq n}(t)=\frac{N_{a b(\neq n)}(t)}{N_{\text {ress }(\neq n)}}$

$(\neq 1,2,3, \ldots, n)$ denotes a cell.

$$
\begin{aligned}
& \left(x_{k+1}\right)_{z_{1}}= \begin{cases}S_{z_{1}}\left(\theta_{k} x_{k}+w_{k}\right)_{z_{1}}, & 0 \leq \rho_{z_{1}}<1 \\
0, & \rho_{z_{1}}=0\end{cases} \\
& \left(x_{k+1}\right)_{z_{2}}= \begin{cases}S_{z_{2}}\left(\theta_{k} x_{k}+w_{k}\right)_{z_{2}}, & 0 \leq \rho_{z_{1}}<1 \\
0, & \rho_{z_{1}}=0\end{cases} \\
& \left(x_{k+1}\right)_{z_{3}}= \begin{cases}S_{z_{3}}\left(\theta_{k} x_{k}+w_{k}\right)_{z_{3}}, & 0 \leq \rho_{z_{3}}<1 \\
0, & \rho_{z_{3}}=0\end{cases} \\
& \left(x_{k+1}\right)_{z_{4}}= \begin{cases}S_{z_{4}}\left(\theta_{k} x_{k}+w_{k}\right)_{z_{4}}, & 0 \leq \rho_{z_{4}}<1 \\
0, & \rho_{z_{4}}=0\end{cases}
\end{aligned}
$$

Meaning :

- 0 no future position in the zone $Z$.

- 1 Major destination resource area is fully occupied and a subscriber in this area will be unable to communicate the unpleasant to the user and penalize the operator.

With inclusion of the density parameter, the operator will not only predict the future movements of the subscriber, but also see if the destination area is saturated or not in terms of resources telecom. In the case of the saturated zone of the operator will be able to add resources to meet the needs of these customers.

\section{Result and Discussion}

Model parameters and programming tools with parameters of the model GSM/GPRS.

$>$ The study is the exploitation of traces collected from operators of Morocco.

Transition period values generated by the exploitation of network traces, tested in this model (slots for one day $24 \mathrm{~h}$ ).

\section{Probability Parameters}

$>$ Real traces: halting probability and transition (one day, 150,000 subscribers) areas: estimation by google earth (Figure 4 ).

\section{Simulation and Programming Tools}

Mobility modeling: Microsoft Excel 2010, Matlab 2010, Google

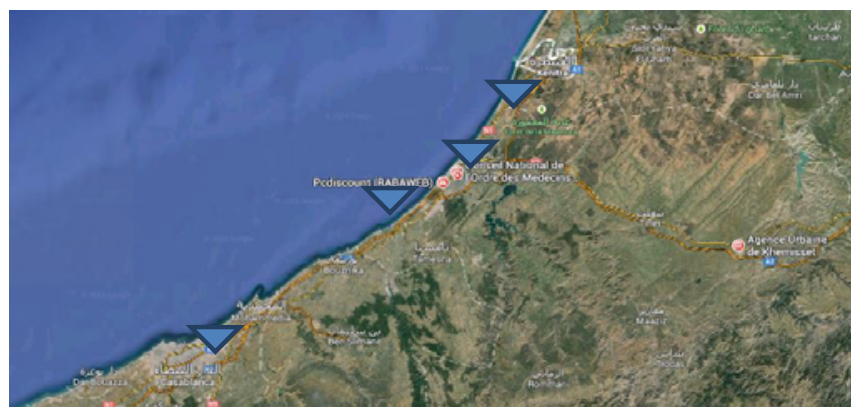

Figure 4: Probability estimation by google earth.

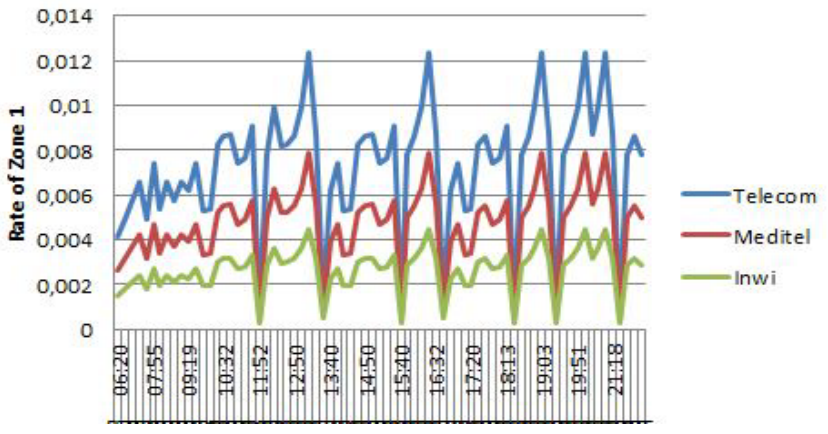

Figure 5: Statistical Area $1\left(z_{1}\right)$ Operator 1, Operator 2 and Operator 3.

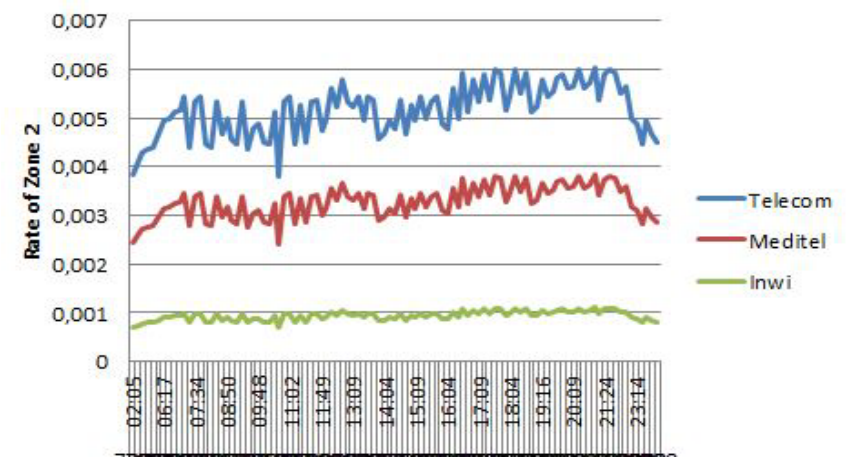

Figure 6: Statistical Area $2\left(z_{2}\right)$ of Operator 1, Operator 2 and Operator 3

Earth Pro v4.01.

$>$ Matlab (soufgarde) $\rightarrow$ text file (input) $\rightarrow$ google earth, Ms Excel (2010).

\section{Deterministic Method}

We calculated the statistics of subscribers in the Kenitra-SaleRabat-Casa train spending $24 \mathrm{~h}$ of those Telecom operators in Morocco (Operator 1, Operator 2 and Operator 3) (Figure 5-8).

Where the density is greater, we need to add resources telecom to meet the needs of future subscribers.

\section{Conclusion}

We study a deterministic Mobility (subscribers in a cell in a train) by introducing a matrix of time slots of this model with the actual 
Citation: Allami J, Ez-Zahraouy H, Benyoussef A (2015) Traffic Network and Optimization a Future Subscriber's Mobile Telecom Operator in Train. J Generalized Lie Theory Appl 9: 219. doi: 10.4172/1736-4337.1000219

Page 4 of 4

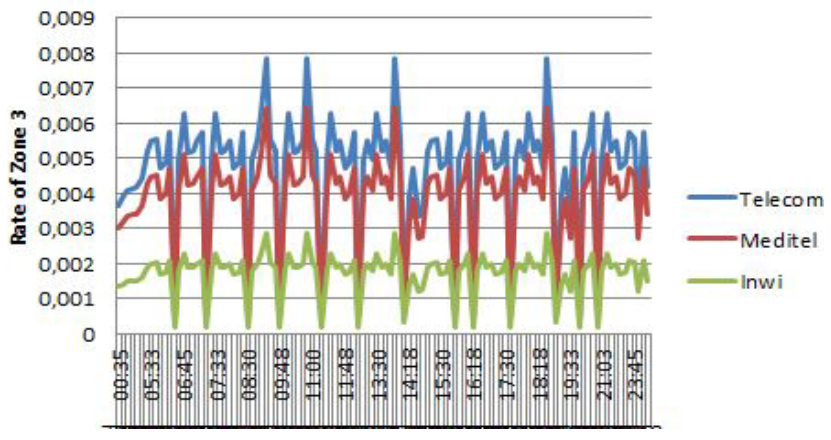

Figure 7: Statistical Area $3\left(z_{3}\right)$ of Operator 1, Operator 2 and Operator 3.

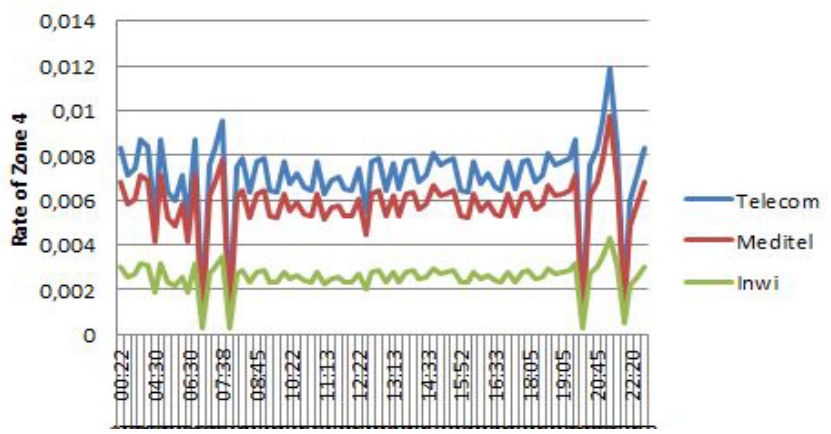

Figure 8: Statistical Area 4( $\left.z_{4}\right)$ Operator 1, Operator 2 and Operator 3.

calculated probability of stationarity and transition are taken into account the number of subscribers, speed, time of association and movement stages for optimization and future planning of a cellular network by the technique of centroid (coordinate system) which gives a good prediction of the future position of Morocco operator : Inwi, Meditel and Telecom with integration a density of actual traces of GSM, GPRS, UMTS, WIMAX..., which leads us to predict a future telecom subscribers and control the future data telecom mobile signal and the operator will predict the future movement of the subscribers and will see if the destination area is saturated or not in terms of resources telecom for add resources to meet the needs of future subscribers.

\section{References}

1. Zonoozi MM, Dassanayake $P$ (1997) User mobility modeling and characterization of mobility patterns. IEEE Journal on Selected Areas in Communications 15: 1239-1252.

2. Bratanov PI, Bonek E (2003) Mobility Model of Vehicle-Borne Terminals in Urban Cellular Systems. IEEE transactions on vehicular technology 52: 947952

3. Hassan H (2006) Multimedia Traffic Modeling and Performance Analysis in Heterogeneous Networks. University of Paul Sabatier - Toulouselll, EDSYS, French.

4. Kalden R (2003/04) Mobility Management in GPRS, Seminar Data communication and Distributed System, Ericsson Research, corporate Unit, Ericsson Eurolab Deutschland $\mathrm{GmbH}$.

5. Leland WE, Taqqu MS, Willinger W, Wilson DV (1994) On the self similarity nature of Ethernet traffic. IEEE/ACM transaction on networking 2: 1-15.

6. Kulavaratharasah MD, Aghvami AH (1999) Teletraffic Performance Evaluation of Microcellular Personal Communication Networks (PCN's) with Prioritized Handoff Procedures", IEEE Transactions on Vehicular Technology 48: 137-152.
7. Wolf DE, Schreckenberg M, Bachem A (1996) Traffic and granular flow, World scientific, Singapore.

8. Wolfram S (1986) Theory and applications of cellular automata, World Scientific, Singapore.

9. Ez-Zahraouy H, Benyoussef A (2008) Eur Phys JB.

10. Nagel K, Schrenckenberg M (1992) J Phys I (France) 2: 2221.

11. Schadchneider A, Schreckenberg M (1993) J Phys A 26: 1679.

12. Nagatani T (1993) J Phys A 26: 11015.

13. Boccara N, Fuks H, Zeng Q (1997) J Phys A 30: 3329

14. Benjamin SC, Johns NF, Hui PM (1996) J Phys A 29: 3119

15. Nagatani T (1993) Phys Rev E 48: 3290.

16. Nagatani T (1996) Physica A 233: 137.

17. Dahmouni H, Morin B, Vaton S (2005) Performance Modeling of GSM/GPRS Cells with Different Radio Resource Allocation Strategies. IEEE WCNC 3: 1317-1322.

18. Dahmouni H, Rosse D, Morin B, Vaton S (2005) Analytical Model for performance Evaluation of GPRS/EDGE Multi-service Networks, 7th IFIP International Conference on Mobile and Wireless Communications Networks, Marrakech, Morocco.

19. Allami J, Ez-Zahraouy H, Benyoussef A (2104) Traffic Network and Antenna Telecom Mobile with a Tramway. International Journal of Recent Technology and Engineering 3: 60-64.

20. Ayari A, Ayadi M, Khedher H, Tabbane S (2010) New method for cellular network traces exploitation, The Sixth International Conference on Networking and Services, Cancun, Mexico.

21. Ayari A, Ayadi M, Khedher H, Ez-Zahraouy H, Tabbane S (2009) Modeling of cellular network subscriber mobility, the fifth advanced international conference on telecommunication, Venice/Mestre, Italy.

22. Ayari A, Ayadi M, Khedher $\mathrm{H}$, Ez-Zahraouy H, Tabbane $\mathrm{S}$ (2009) Probabilistic mobility modeling for cellular network subscriber, Communication Society, Vehicular Technology, Information Theory and Aerospace and Electronics Systems Technology, Wireless Communications and Networking, Aalborg Congress and Culture Centre, Aalborg, Denmark.

23. Ez-Zahraouy H, Benrihane Z, Benyoussef A (2005) Int J Mod Phys C 16: 1461

24. Ez-Zahraouy H, Benrihane Z, Benyoussef A (2004) Int J Mod Phys.

25. Ez-Zahraouy H, Benrihane Z, Benyoussef A (2004) Eur Phys J.

26. Benyoussef A, Chakib H, Ez-Zahraouy H (2003) Phys Rev.

27. Derrida B, Domany E, Mukamel D (1992) J Stat Phys.

28. Mierswa I, Wurst M, Klinkenberg R, Scholz M, Euler T (2006) YALE: Rapid Prototyping for Complex Data Mining Tasks, in Proceedings of the 12th ACM SIGKDD International Conference on Knowledge Discovery and Data Mining.

29. Breiman L, Friedman J, Olshen R, Stone CJ (1984) Classification and Regression Trees, Wadsworth International.

30. Quinlan R (1993) C4.5: Programs for Machine Learning, Morgan Kaufmann Publishers Inc. San Francisco, CA, USA.

31. Daniel T, Larose T, Vallaud (2005) Data to Knowledge: An introduction to data mining, Vuibert.

32. Zighed D, Rakotomalala R (2000) Graphs and Data Mining Induction --Apprentissage, Hermes.

33. Skowron A, Rauszer C (1992) The discernability matrices and functions in information systems. In intelligent decision support: Handbook of applications and advances of Rough Sets theory. Springer Netherlands.

34. Slowinski R (1992) Handbook of Applications and Advances of the Rough Sets Theory, intelligent decision support. Springer Netherlands. 